\title{
Disease of Inclusion Bodies in a Boa Constrictor (Boa constrictor constrictor) Diagnosed by Histopatology and Electronic Microscopy.
}

\author{
Méndez, Adriana $^{1 *}$, Ramos, Pilar ${ }^{2}$, Patiño, Valeria ${ }^{1}$, Saravia, Melissa ${ }^{1}$. \\ ${ }^{1}$ Universidad Nacional Autónoma de México, Facultad de Medicina Veterinaria y Zootecnia, \\ Departamento de Patología, CDMX, México. \\ ${ }^{2}$ Instituto Nacional de Cancerología, Patología, CDMX, México \\ *Corresponding author:mvzadrimb@gmail.com.
}

The disease of inclusion bodies (DIB) is a common entity in the Boa constrictor, as well as in other types of boas and pythons. It was first identified in 1970 in the United States, since then it has been reported in Europe, Australia and Africa, mainly affecting snakes from private and zoological collections [1]. The distribution is worldwide because it occurs in captive animals and there are no reports in wild animals. It is a fatal infectious disease caused by an organism resembling a retrovirus (retrovirus - like organism). In recent literature it has been suggested that this disease is caused by a Reptarenavirus, wrapped, segmented, single-stranded RNA. The clinical signs are varied, including anorexia, regurgitation, incoordination, tremor, opistotones, stomatitis and the presence of some proliferative processes. However, it can occur subclinically, extending from weeks to months. Animals infested with mites, Ophionyssus natricis, tend to be more susceptible, since it is thought that this parasite may be the virus vector. It is also speculated that mice and / or rats used to feed the boas can be a source of infection, however, it has not been proven. The incubation period is unknown and death is usually caused by secondary bacterial, parasitic or fungal infections. There are currently no treatments and vaccines available. The recommendation for animals infected by this virus, is the application of euthanasia, to prevent transmission to the congeners [2].

The most commonly reported macroscopic lesions are atrophy and fibrosis of the spleen, ulcerative stomatitis, nodular lesions in the stomach and esophagus. Snakes diagnosed with DIB usually present with concomitant neoplasia, the relationship between the infectious and neoplasic process has not been elucidated. It is characterized by the formation of eosinophilic to amphiphilic intracytoplasmic inclusion bodies, of variable sizes. Inclusion bodies can be seen in blood cells, myeloid precursors in the bone marrow and in practically all tissues, especially neurons of the central nervous system, liver, stomach, esophageal tonsils and pancreas. In the case of pythons, inclusion bodies are more common in neurons and glial cells with or without associated inflammation [3]. The apparent absence of inclusion bodies does not indicate that the animal is not being affected by the virus. Sometimes it is necessary to use complementary techniques to be able to discard it. In transmission electron microscopy, inclusions can be observed, which consist of a $64 \mathrm{kDa}$ nucleoprotein deposited by surrounding radiodense polyribosomes [4].

Boa constrictor constrictor, an eight-year-old male, with a history of anorexia, posterior third paralysis, tremors in masseter and coprostasis, is referred for postmortem study to the FMVZ - UNAM pathology department. In the macroscopic study it was observed that the esophagus was dilated and the stomach was thickened. The liver was reddish brown and when dissecting blood came out. Microscopically, the lung, liver, spleen and brain presented eosinophilic intracytoplasmic inclusion bodies (Fig 1). Additionally, transmission electron microscopy was performed in which electrodense viral particles were found whose diameters ranged from 70 to $80 \mathrm{~nm}$. Likewise, clusters of ecetrodense material compatible with lipofucsin were observed (Fig. 2). 
Conclusion: disease by inclusion bodies is the most common infectious disease in snakes. The diagnosis is made mainly by histopathology, ante and post mortem, through the observation of eosinophilic intranuclear inclusion bodies in H\&E staining, which could be observed in this case, in neurons and epithelial cells of various organs. In this case the use of transmission electron microscopy to identify nucleoprotein or dense bodies, confirming the disease.
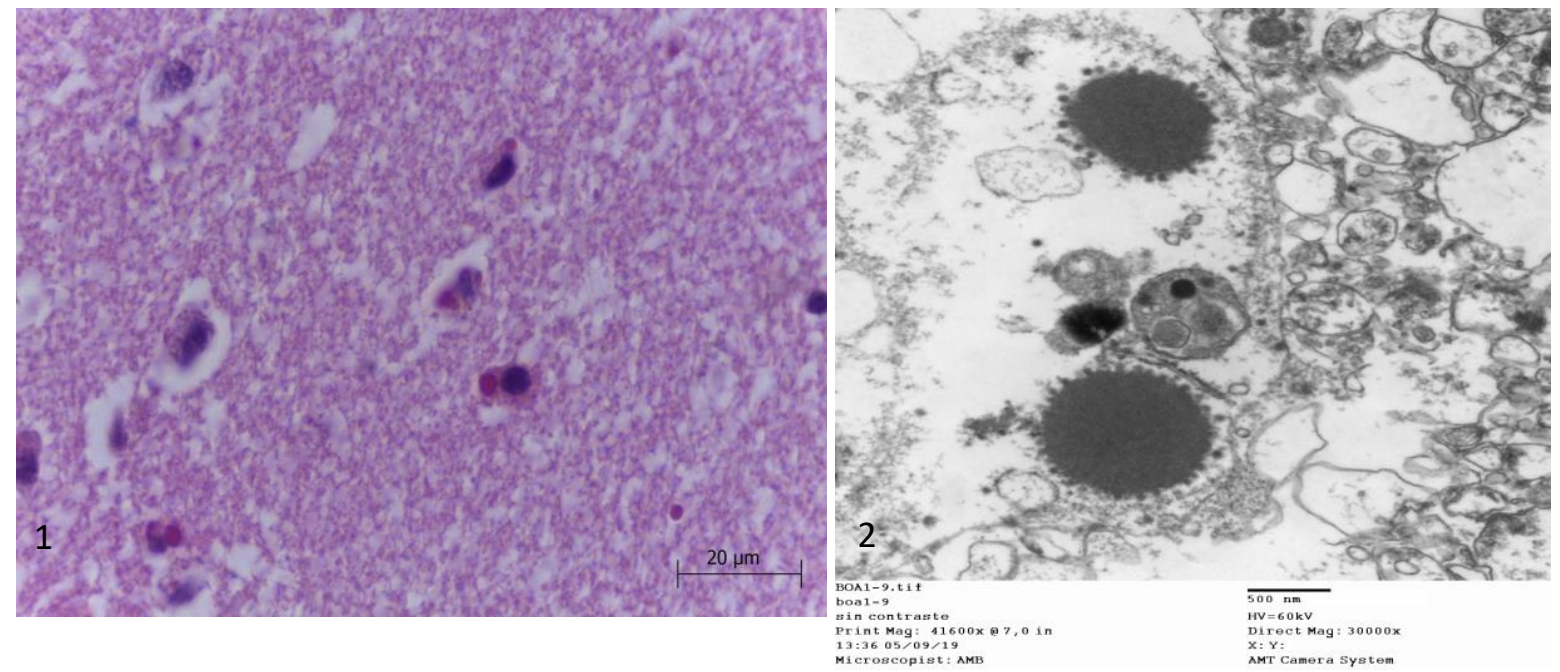

Figure 1. Histologic photomicrograph of the brain of a Boa constrictor. Observe in the cytoplasm of a glial cell a body of eosinophilic intracytoplasmic inclusion. H\&E staining. 20 micron bars. Figure 2. Transmission electronic photography. Observe in the neuropil electrodense structures surrounded by electrodense viral particles measuring 70 to $80 \mathrm{~nm}$ in diameter. Contrast technique with uranyl acetate and lead citrate. $500 \mathrm{~nm}$ bars.

\section{References:}

[1] Jacobson ER, In: Jacobson E Infectious disease and pathology of reptiles, (2017), p.410 - 411, 450, $452,457$.

[2] Jacobson ER et al, In: Jacobson E Infectious disease and pathology of reptiles, (2007), p.339 - 340.

[3] Jacobson ER et al, Am J Vet Res, 62, (2001) p. 217 - 224.

[4] MacLachlan NJ et al, In: Fenner's Veterinary Virology. 4th ed, (2017), p.434. 